\title{
SENSIBILIDAD MICROBIANA DE ESCHERICHIA COLI EN INFECCIONES URINARIAS EXTRAHOSPITALARIAS
}

\author{
J.M. SÁNCHEZ MERINO, C. GUILLÁN MAQUIEIRA*, C. FUSTER FOZ**, \\ F.J. MADRID GARCİA, M. JIMÉNEZ RODRÍGUEZ***, J. GARCÍA ALONSO
}

\begin{abstract}
Servicio de Urología. *Servicio de Ginecología. **Servicio de Microbiología. ${ }^{* * *}$ Medicina Preventiva.
\end{abstract} Hospital del Bierzo. Ponferrada. León.

Actas Urol Esp. 27 (10): 783-787, 2003

\section{RESUMEN}

SENSIBILIDAD MICROBIANA DE ESCHERICHIA COLI EN INFECCIONES URINARIAS EXTRAHOSPITALARIAS

OBJETIVO: El tratamiento empírico de las infecciones urinarias extrahospitalarias debe tener en cuenta la sensibilidad de las bacterias potencialmente causantes de las mismas. Además, la comparación con años anteriores permite observar la evolución de la sensibilidad microbiana. Analizamos estos aspectos con respecto a Escherichia coli en nuestro medio.

MATERIAL Y MÉTODOS: Se analizaron los urocultivos positivos para Escherichia coli obtenidos de muestras enviadas desde atención primaria en El Bierzo (León) durante los años 2002 y 1998, en número de 895 y 595 , respectivamente. Se determinó la sensibilidad de este germen a nueve antibióticos de uso frecuente. Se analizó mediante chi cuadrado la existencia de diferencias estadísticamente significativas de sensibilidad en los años estudiados.

RESULTADOS: El 63,4\% de todos los urocultivos positivos de atención primaria en 2002 y el 50,8\% en 1998 resultaron positivos para Escherichia coli. La sensibilidad in vitro en 2002 y 1998, respectivamente, ha sido la siguiente: fosfomicina $(99,2 \%-99,3 \%$; $\mathrm{p}=\mathrm{NS}$ *), cefixima $(98,3 \%-92,9 \%$; $<<0,001)$, cefuroxima $(96,5 \%-94,1 \%$; $<<0,05)$, nitrofurantoína (94,5\%-86,9\%; p<0,001), amoxicilina-clavulánico (93,1\%-90,1\%; p<0,05), ciprofloxacino (77,1\%-81,6\%; p<0,05), norfloxacino $\left(75,8 \%-80,3 \%\right.$; p <0,05), cotrimoxazol $(71,5 \%-73,4 \%$; $\mathrm{p}=\mathrm{NS} *)$ y ampicilina $(44 \%-41,4 \%$; $\mathrm{p}=\mathrm{NS} *)$. ( ${ }^{*} \mathrm{NS}=$ No significativo).

CONCLUSIONES: El conocimiento de los patrones de sensibilidad de los gérmenes más frecuentemente aislados en muestras de orina en cada zona permite aplicar el tratamiento empírico más adecuado en caso de infección.

PALABRAS CLAVE: Infección del tracto urinario. Sensibilidad microbiana. Infección extrahospitalaria.

\section{ABSTRACT}

\section{ANTIMICROBIAL SUSCEPTIBILITY OF ESCHERICHIA COLI ASSOCIATED WITH COMMUNITY-ACQUIRED URINARY} TRACT INFECTIONS

OBJECTIVE: For effective empiric therapy of urinary tract infections in the extrahospital setting the susceptibility pattern of uropathogens should be considered. Moreover, the evolution in sensitivity can be observed when comparing with susceptibility patterns in the previous years. This paper presents an analysis of our experience with Escherichia coli.

MATERIAL AND METHODS: During 2002 and 1998, 895 and 595 strains of Escherichia coli respectively, isolated from extrahospitalary bacteriurias were collected in ten health centers in Bierzo (León, Spain). Sensitivity to nine most commonly antibiotics used in the clinical practise was determined. The existence of significant differences of susceptibility among years (2002-1998) was analyzed by the chi square test.

RESULTS: Escherichia coli accounted for $63.4 \%$ of all isolates in 2002 and $50.8 \%$ in 1998. The prevalence of in-vitro susceptibilities to antibiotics were (2002-1998): fosfomycin (99.2\%-99.3\%; p=NS*), cefixime (98.3\%-92.9\%; p<0.001), cefuroxime (96.5\%-94.1\%; p<0.05), nitrofurantoin (94.5\%-86.9\%; p<0.001), amoxycillin-clavulanic acid $(93.1 \%-90.1 \%$; $\mathrm{p}<0.05)$, ciprofloxacin $(77.1 \%-81.6 \%$; p<0.05), norfloxacin $(75.8 \%-80.3 \%$; $<<0.05)$, cotrimoxazole $(71.5 \%-73.4 \%$; $\mathrm{p}=\mathrm{NS} *)$ and ampicillin (44\%-41.4\%; $\mathrm{p}=\mathrm{NS} *)$. ( ${ }^{\mathrm{NS}}=$ No significant differences).

CONCLUSION: The knowledge of the sensitivity of uropathogens to antimicrobians in a specific medium can allow us to use antibiotics rationally and initiate empirical therapy.

KEY WORDS: Urinary tract infection. Antibiotic susceptibility. Community-acquired infection. 
$\mathrm{L}^{\mathrm{a}}$ a importancia de las infecciones urinarias reside en el enorme impacto que representa en la población, constituyendo del 2 al 5\% de las consultas de atención primaria ${ }^{1}$. Escherichia coli es su causa más común, aunque su frecuencia concreta varía según el tipo de pacientes ${ }^{2}$.

La resistencia bacteriana de Escherichia coli a los antibióticos se relaciona con el consumo de éstos ya que, la presión selectiva que ejercen, favorece la creación, adaptación y diseminación de mecanismos de resistencia a los antimicrobianos. La prevalencia creciente de resistencias hace imprescindible que para orientar racionalmente el tratamiento empírico de la infección urinaria en el medio extrahospitalario, lo que constituye una práctica habitual y recomendada ${ }^{3}$, los laboratorios de microbiología informen periódicamente a los clínicos de los patrones de sensibilidad de las bacterias potencialmente causantes de infección en cada área geográfica ${ }^{2,4}$.

El propósito de este estudio es evaluar la sensibilidad a varios antibióticos orales en cepas de Escherichia coli aisladas en muestras de orina de pacientes de atención primaria en la Comarca del Bierzo (León) en el año 2002 y detectar las variaciones de sensibilidad en los últimos años, comparando los patrones de sensibilidad con los obtenidos en 1998.

\section{MATERIAL Y MÉTODOS}

Se realizó un estudio retrospectivo de los urocultivos positivos para Escherichia coli obtenidos de muestras enviadas al Laboratorio de Microbiología del Área de Salud del Bierzo (León) desde los diez centros de atención primaria del área, que atienden en su conjunto una población aproximada de 150.000 habitantes. Los urocultivos analizados corresponden a la totalidad de los que resultaron positivos para Escherichia coli (895 en el año 2002 y 595 en 1998), procedentes de población urbana y rural extrahospitalaria con diagnóstico de sospecha de infección urinaria y/o control postratamiento.

Se estudió la sensibilidad in vitro de Escherichia coli a nueve antibióticos de uso frecuente en la práctica clínica: las fluoroquinolonas norfloxacino y ciprofloxacino, los betalactámicos amoxicilina-clavulánico, ampicilina y las cefalosporinas cefuroxima ( $2^{\mathrm{a}}$ generación) y cefi- xima ( $3^{\text {a }}$ generación), fosfomicina, nitrofurantoína y cotrimoxazol. Se utilizó el medio de cultivo de Mueller-Hinton. Se determinaron las concentraciones mínimas inhibitorias mediante el sistema MicroScan ${ }^{\circledR}$ (Dade Behring). En la interpretación de la sensibilidad en el año 2002 se aplicaron los criterios del NCCLS (National Committe for Clinical Laboratory Standards) de 2001, que no han variado con respecto a los utilizados en el año $1998^{5,6}$ (Tabla I).

\section{TABLA I}

VALORES DE LA CONCENTRACIÓN MÍNIMA INHIBITORIA PARA LA INTERPRETACIÓN DE LA SENSIBILIDAD, SEGÚN LA NCCLS ${ }^{5,6}$

\begin{tabular}{|l|c|}
\hline \hline Antimicrobiano & Sensible \\
\hline Fosfomicina & $\leq 64$ \\
\hline Cefixima & $\leq 1$ \\
\hline Cefuroxima & $\leq 8$ \\
\hline Nitrofurantoína & $\leq 32$ \\
\hline Amoxicilina-clavulánico & $\leq 8 / 4$ \\
\hline Ciprofloxacino & $\leq 1$ \\
\hline Norfloxacino & $\leq 4$ \\
\hline Cotrimoxazol & $\leq 2 / 38$ \\
\hline Ampicilina & $\leq 8$ \\
\hline
\end{tabular}

Valores expresados en $\mu \mathrm{g} / \mathrm{mL}$

Se aplicó la prueba de chi cuadrado para valorar si existían diferencias significativas $(p<0,05)$ de sensibilidad de Escherichia coli a los antibióticos estudiados en los años 2002 y 1998. En la comparación se excluyeron las cepas de sensibilidad intermedia.

\section{RESULTADOS}

Se obtuvo el crecimiento de Escherichia coli en el $63,4 \%$ y el 50,8\% de la totalidad de los urocultivos enviados desde atención primaria que resultaron positivos en el año 2002 y 1998, respectivamente.

La comparación de la sensibilidad in vitro de Escherichia coli entre los años 2002 y 1998 a los antibióticos estudiados se resume en la Tabla II. La mejor sensibilidad frente a Escherichia coli la presentó la fosfomicina, por encima del 99\%, sin apenas variación desde 1998. Por otra parte, 
Escherichia coli no sólo mantuvo una buena sensibilidad a los betalactámicos cefixima $(98,3 \%)$, cefuroxima $(96,5 \%)$ y amoxicilina-clavulánico $(93,1 \%)$, sino que además se ha incrementado de manera estadísticamente significativa con respecto a 1998 (más de cinco puntos porcentuales para cefixima, más de dos para cefuroxima y tres para amoxicilina-clavulánico). Similar comportamiento mostró la nitrofurantoína, con una mejor sensibilidad, desde aproximadamente el $87 \%$ en 1998 a casi el $95 \%$ en $2002(\mathrm{p}<0,001)$.

Por otra parte, Escherichia coli presentó tasas de resistencia a las fluoroquinolonas superiores al $20 \%$. Además, el porcentaje de cepas resistentes ha aumentado de manera estadísticamente significativa en los últimos años. Por último, las sensibilidades para cotrimoxazol y ampicilina se mantuvieron bajas, sin apenas variaciones (por debajo del $73,4 \%$ para el primero y del $44 \%$ para la segunda).

\section{DISCUSIÓN}

El tratamiento empírico de las infecciones urinarias es una práctica habitual en el medio extrahospitalario ${ }^{3}$. Sin embargo, es necesario tener en cuenta los patrones de sensibilidad de las bacterias potencialmente causantes de las mismas. Estos patrones pueden variar entre distintas zonas e incluso en una misma área geográfica con el paso del tiempo ${ }^{4,7}$.
Escherichia coli ha sido el germen más frecuentemente aislado en muestras de orina de pacientes de atención primaria en la Comarca del Bierzo. La distribución de los microorganismos en el medio extrahospitalario en trabajos recientes en nuestro país es similar, con predominio de Escherichia coli $(63-82,1 \%)^{2,8,9}$. Por tanto, el conocimiento del patrón de sensibilidad antibiótica de éste germen es fundamental para orientar adecuadamente el tratamiento empírico de la infección urinaria en nuestro medio.

Mientras que los microorganismos no difieren sensiblemente de unas zonas a otras en los países desarrollados, sí lo hacen las resistencias a los diferentes antimicrobianos. En nuestro estudio la fosfomicina mostró una excelente actividad in vitro frente a Escherichia coli, con menos del uno por ciento de cepas resistentes. Diversos estudios han demostrado una sensibilidad entre el 95,7\% y $99,5 \%{ }^{8,10,11}$. Este aspecto, junto con la infrecuencia de efectos adversos y las concentraciones urinarias alcanzadas, más altas que la concentración mínima inhibitoria para los patógenos del tracto urinario, convierten la fosfomicina trometamol en un excelente antibiótico para el tratamiento de la infección urinaria no complicada ${ }^{11}$.

Por otra parte, los porcentajes de resistencias a las cefalosporinas cefixima y cefuroxima, la nitrofurantoína y la amoxicilina-clavulánico fueron bajos, con disminución con respecto a 1998 (Tabla II). En general, la sensibilidad mostrada

\section{TABLA II}

COMPARACIÓN DE LA SENSIBILIDAD MICROBIANA DE ESCHERICHIA COLI EN LA COMARCA DEL BIERZO EN LOS AÑOS 2002 Y 1998

\begin{tabular}{|c|c|c|c|c|c|c|c|}
\hline & \multicolumn{3}{|c|}{2002} & \multicolumn{3}{|c|}{1998} & \multirow[b]{2}{*}{$\mathbf{p}$} \\
\hline & $\mathbf{N}$ & Sensibles & $\%$ & $\mathbf{N}$ & Sensibles & $\%$ & \\
\hline Fosfomicina & 891 & 884 & 99,2 & 595 & 591 & 99,3 & NS \\
\hline Cefixima & 891 & 876 & 98,3 & 309 & 287 & 92,9 & $\mathrm{p}<0,001$ \\
\hline Cefuroxima & 892 & 861 & 96,5 & 591 & 556 & 94,1 & $\mathrm{p}<0,05$ \\
\hline Nitrofurantoína & 894 & 845 & 94,5 & 595 & 517 & 86,9 & $\mathrm{p}<0,001$ \\
\hline Amoxicilina-clavulánico & 895 & 833 & 93,1 & 595 & 536 & 90,1 & $\mathrm{p}<0,05$ \\
\hline Ciprofloxacino & 895 & 690 & 77,1 & 592 & 483 & 81,6 & $\mathrm{p}<0,05$ \\
\hline Norfloxacino & 894 & 678 & 75,8 & 595 & 478 & 80,3 & $\mathrm{p}<0,05$ \\
\hline Cotrimoxazol & 892 & 638 & 71,5 & 595 & 437 & 73,4 & NS \\
\hline Ampicilina & 895 & 394 & 44 & 590 & 244 & 41,4 & NS \\
\hline
\end{tabular}

N: número de urocultivos en los que se probó la sensibilidad al antibiótico. NS: no significativo. 
por Escherichia coli para estos antibióticos en nuestro medio está en consonancia con la reflejada en la literatura: 97,8\%-100\% para cefixima, 95,8\%-96,8\% para nitrofurantoína y 95,8\%$99,2 \%$ para amoxicilina-ácido clavulánico ${ }^{8,10}$. Los dos últimos presentan unas cifras discretamente inferiores en nuestro estudio, probablemente debido a que no se han estratificado los resultados según las características clínicas de los pacientes (edad, sexo, tipo de patología, control postratamiento).

Los porcentajes de sensibilidad a ciprofloxacino en nuestro estudio son similares a los publicados por Queipo et al. ${ }^{8}$ y Kahlmeter ${ }^{10}(69,6 \%$ $84,2 \%$ para norfloxacino y $71,7 \%-85,3 \%$ para ciprofloxacino) en estudios realizados en mujeres con infecciones urinarias no complicadas adquiridas en la comunidad en nuestro país. Por otra parte, con las dos fluoroquinolonas se constató un aumento de las resistencias en relación a 1998. Este incremento ya es conocido en España. Así, Queipo et al. ${ }^{2}$ detectaron una progresión en la resistencia bacteriana de Escherichia coli desde el 3\% en 1990 al 23\% en 1998. De cualquier manera, las cifras de resistencia a quinolonas en España superan ampliamente las comunicadas en otros países europeos y EE.UU. ${ }^{10,12}$.

Escherichia coli ha mostrado una baja sensibilidad a cotrimoxazol y ampicilina, que por otro lado permanece estable en España con el paso de los años ${ }^{4,7}$, aunque con frecuencias inferiores a las comunicadas en otros países. Así, Kahlmeter ${ }^{10}$ en un estudio multinacional obtiene tasas de resistencia del $25,7 \%$ para cotrimoxazol y $53,9 \%$ para ampicilina en España, cifras muy similares a las obtenidas en Portugal. En el resto de Europa y Canadá las resistencias oscilan entre 5,5\%$22,5 \%$ para cotrimoxazol y $15,5 \%-44,8 \%$ para ampicilina.

En nuestro país las cifras de resistencias a las quinolonas se disparan debido a su uso masivo en la práctica clínica ${ }^{13,14}$. Su amplio espectro de acción, potencia bactericida, propiedades farmacocinéticas (buena biodisponibilidad oral, baja unión a proteínas plasmáticas y eliminación renal) y su buena tolerancia las han convertido en uno de los grupos de antibióticos de más amplia aceptación ${ }^{2}$. También se ha implicado en esta elevada tasa de resistencias la ingesta de pequeñas dosis de antimicrobianos dentro de la cadena alimentaria por consumo de carne procedente de animales tratados con antibióticos ${ }^{2,15}$.

Por otra parte, algunos de los antimicrobianos usados específicamente para tratar las infecciones urinarias adquiridas en la comunidad, como la fosfomicina y la nitrofurantoína, todavía presentan niveles sorprendentemente bajos de resistencias, a pesar de los años de uso. Una explicación plausible podría ser el hecho de que no se utilizan, en general, en los hospitales ${ }^{9}$.

Aunque nuestro estudio no tuvo en cuenta las características clínicas de los pacientes, los patrones de sensibilidad obtenidos son similares a los encontrados en otros realizados en el ámbito extrahospitalario. De esta manera, parece razonable utilizar los datos proporcionados por los laboratorios de microbiología como orientación para el tratamiento empírico de las infecciones urinarias, en este caso en el paciente ambulatorio. No obstante, la mejor aproximación se obtendría estratificando los resultados por edad, sexo y tipo de patología.

En resumen, los antibióticos que presentaron menores índices de resistencias en nuestro medio fueron la fosfomicina, cefixima y cefuroxima, seguidos por nitrofurantoína y amoxicilina-clavulánico. Debe reconsiderarse el uso de ciprofloxacino, norfloxacino, cotrimoxazol y ampicilina en el tratamiento empírico de las infecciones de orina extrahospitalarias. Estos resultados son válidos para todo el periodo de estudio y son similares a los obtenidos en otras áreas geográficas de nuestro entorno.

\section{REFERENCIAS}

1. JIMÉNEZ M, FERNÁNDEZ E.: Infecciones urinarias en la mujer y en el paciente geriátrico. En: Navío S, editor. Patología urológica infecciosa. Madrid. Aula Médica, Ediciones 1999: 87-102.

2. QUEIPO JA, BUDÍA A, JIMÉNEZ MJ et al.: Evolución de la resistencia microbiana a fluorquinolonas en un hospital terciario. Actas Urol Esp 2000; 24 (5): 381-387.

3. KRIEGER JN.: Urinary tract infections: What's new?. J Urol 2002; 168: 2351-2358.

4. ALÓS JI, GÓMEZ-GARCÉS JL, GARCÍA-BERMEJO I, GARCÍA-GÓMEZ JJ, GONZÁLEZ-PALACIOS R, PADILLA B.: Prevalencia de susceptibilidad de Escherichia coli a quinolonas y otros antibióticos en bacteriurias extrahospitalarias de Madrid. Med Clin (Barc) 1993; 101: 87-90. 
5. NCCLS.: Performance standards for antimicrobial susceptibility testing: eleventh informational supplement. NCCLS document M100-S11 (ISBN 156238-426-0). NCCLS, 940 West Valley Road, Suite 1400; Wayne, Pennsylvania 19087-1898, USA 2001.

6. NCCLS.: Methods for dilution antimicrobial susceptibility tests for bacteria that grow aerobically fourth edition; approved standard. NCCLS document M7-A4 (ISBN 1-56238-309-4). NCCLS, 940 West Valley Road, Suite 1400; Wayne, Pennsylvania 19087, USA 1997.

7. ALOS JI, BALAS D, GÓMEZ JL, GRUPO DE ESTUDIO DE INFECCIÓN EN ATENCIÓN PRIMARIA. Prevalencia de susceptibilidad a quinolonas y otros antibióticos en microorganismos aislados de bacteriurias extrahospitalarias de Madrid en 1995. Rev Clín Esp 1997; 197: 167-171.

8. QUEIPO JA, GIMÉNEZ MJ, DIOSDADO N, GÓMEZ-FERRER A, GOBERNADO M, JIMÉNEZ JF.: Cistitis aguda en la mujer. Sensibilidad microbiana actual en nuestro medio. Actas Urol Esp 2001; 25 (8): 567-572.

9. KAHLMETER G.: The ECO-SENS Project: a prospective, multinational, multicentre epidemiological survey of the prevalence and antimicrobial susceptibility of urinary tract pathogens-interim report. $J$ Antimicrob Chemother 2000; 46 (Suppl S1): 15-22.

10. KAHLMETER G.: An international survey of the antimicrobial susceptibility of pathogens from uncomplicated urinary tract infections: The ECOSENS Project. J Antimicrob Chemother 2003; 51: 69-76.

11. GARCÍA-RODRÍGUEZ JA, TRUJILLANO I, BAQUE$\mathrm{RO} F$ et al.: In vitro activity of fosfomycin trometamol against pathogens from urinary tract infections: a Spanish multicenter study. $J$ Chemother 1997; 9 (6): 394-402.

12. GUPTA K, SCHOLES D, STAMM WE.: Increasing prevalence of antimicrobial resistance among uropathogens causing acute uncomplicated cystitis in women. JAMA 1999; 281 (8): 736-738.

13. FERNÁNDEZ A, LANTERO M, GASTAÑARES MJ et al.: Escherichia coli resistente a quinolonas en el área sanitaria de un hospital de 650 camas. Actas Urol Esp 1994; 18 (6): 634-638.

14. JIMÉNEZ JF, BROSETA E, GOBERNADO M.: Infección urinaria. Actas Urol Esp 2002; 26 (7): 563-573

15. EL GLOBAL.: La FDA prohíbe unos antibióticos utilizados en la alimentación de pollos. El global 2001 enero 19; sección productos: 14 .

Dr. J.M. Sánchez Merino

C/ Francisco González, 25

24400 Ponferrada (León)

(Trabajo recibido el 10 junio de 2003) 